\title{
ULTRALOGICS AND PROBABILITY MODELS
}

\author{
ROBERT A. HERRMANN
}

(Received 9 December 2000)

\begin{abstract}
We show how nonstandard consequence operators, ultralogics, can generate the general informational content displayed by probability models. In particular, a probability model that predicts that a specific single event will occur and those models that predict that a specific distribution of events will occur.
\end{abstract}

2000 Mathematics Subject Classification. 03H10, 60A99.

1. Introduction. In [3], the theory of nonstandard consequence operators is introduced. Consequence operators, as an informal theory for logical deduction, were introduced by Tarski [6]. There are two such operators investigated, the finite and the general consequence operator. Let $L$ be any nonempty set that represents a language and $\mathscr{P}$ the set-theoretic power set operator.

DEFINITION 1.1. A mapping $C: \mathscr{P}(L) \rightarrow \mathscr{P}(L)$ is a general consequence operator (or closure operator) if for each $X, Y \in \mathscr{P}(L)$

(i) $X \subset C(X)=C(C(X)) \subset L$ and if

(ii) $X \subset Y$, then $C(X) \subset C(Y)$.

A consequence operator $C$ defined on $L$ is said to be finite (finitary, or algebraic) if it satisfies

(iii) $C(X)=\cup\{C(A) \mid A \in F(X)\}$, where $F$ is the finite power set operator.

REMARK 1.2. The above axioms (i), (ii), and (iii) are not independent. Indeed, (i) and (iii) imply (ii).

In [3], the language $L$ and the set of all consequence operators defined on $L$ are encoded and embedded into a standard superstructure $\mathcal{M}=\langle\mathcal{N}, \in,=\rangle$. This standard superstructure is further embedded into a nonstandard and elementary extension ${ }^{*} \mathcal{M}=\left\langle{ }^{*} \mathcal{N}, \in,=\right\rangle$. For convenience, ${ }^{*} \mathcal{M}$ is considered to be a $2^{|\mathcal{M}|}$-saturated enlargement. Then, in the usual constructive manner, ${ }^{*} \mathcal{M}$ is further embedded into the superstructure, the Grundlegend structure, $y=\langle Y, \in,=\rangle$ where, usually, the nonstandard analysis occurs. In all that follows in this article, the Grundlegend superstructure $y$ is altered by adjoining to the construction of $\mu$ a set of atoms that corresponds to the real numbers. This yields a $2^{|\mathcal{M}|}$-saturated enlargement $* \mu_{1}$ and the corresponding extended Grundlegend structure $\mathscr{Y}_{1}[1,2]$.

2. The main result. To indicate the intuitive ordering of any sequence of events, the set $T$ of Kleene styled "tick" marks, with a spacing symbol, is used [5, page 202] as they might be metamathematically abbreviated by symbols for the nonzero natural 
numbers. Let $G \subset L_{1}$ be considered as a fixed description for a source that yields, through application of natural laws or processes, the occurrence of an event described by $E \subset L_{1}$. Further, the statement $E^{\prime} \subset L_{1}$ indicates that the event described within the statement $E$ did not occur. Let $L=\{G\} \cup\left\{E, E^{\prime}\right\} \cup T$. As usual, $G, E$, and $E^{\prime}$ are assumed to contain associated encoded general information [4]. Note that for subsets of $L$, bold notation, such as $\mathbf{G}$, denotes the image of $G$ as it is embedded into $\mu_{1}$.

Theorem 2.1. For the language $L$ and any $p \in \mathbb{R}$ such that $0 \leq p \leq 1$, where $p$ represents a theory predicted (i.e., a priori) probability that an event will occur, there exists an ultrachoice function ${ }^{*} C$ and an ultralogic $P_{p}$ with the following properties:

(1) When $P_{p}$ is applied to $\left.* \mathbf{G}\right\}=\{\mathbf{G}\}$ a hyperfinite set of "events" $\left\{a_{1}, \ldots, a_{n}, \ldots,{ }^{*} a_{v}\right\}$ is obtained such that for any " $n$ " trials, $\left\{a_{1}, \ldots, a_{n}\right\}$ is a finite identified "event" sequence, where each $a_{i}$ determines the labeled event $\mathbf{E}$ or labeled non-event $\mathbf{E}^{\prime}$.

(2) The labeled events in (1) are sequentially determined by ${ }^{*} C$, where $C$ determines a sequence $g_{a p}$ of relative frequencies that converges to $p$.

(3) The sequence of relative frequencies $g_{a p}$ determined by ${ }^{*} C$ gives the appearance of theory dependent random chance.

Proof. All of the objects discussed will be members of an informal superstructure at a rather low level and slightly abbreviated definitions, as also discussed in [1, pages $23,30-31]$, are utilized. As usual $\mathbb{N}$ is the set of all natural numbers including zero, and $\mathbb{N}^{>0}$ the set of all nonzero natural numbers.

Let $A=\left\{a \mid\left(a: \mathbb{N}^{>0} \rightarrow \mathbb{N}\right) \wedge\left(\forall n\left(n \in \mathbb{N}^{>0} \rightarrow(a(1) \leq 1 \wedge 0 \leq a(n+1)-a(n) \leq 1)\right)\right)\right\}$. Note that the special sequences in $A$ are nondecreasing and for each $n \in \mathbb{N}^{>0}, a(n) \leq n$. Obviously $A \neq \varnothing$, for the basic example to be used below, consider the sequence $a(1)=0, a(2)=1, a(3)=1, a(4)=2, a(5)=2, a(6)=3, a(7)=3, a(8)=4, \ldots$ which is a member of $A$. Next consider the most basic representation $Q$ for the nonnegative rational numbers where we do not consider them as equivalence classes. Thus $Q=\left\{(n, m) \mid(m \in \mathbb{N}) \wedge\left(n \in \mathbb{N}^{>0}\right)\right\}$.

For each member of $A$, consider the sequence $g_{a}: \mathbb{N} \rightarrow Q$ defined by $g_{a}(n)=$ $(n, a(n))$. Let $F$ be the set of all such $g_{a}$ as $a \in A$. Consider from the above hypotheses, any $p \in \mathbb{R}$ such that $0 \leq p \leq 1$. We show that for any such $p$ there exists an $a \in A$ and a $g_{a p} \in F$ such that $\lim _{n \rightarrow \infty} \mathcal{g}_{a p}(n)=p$. For each $n \in \mathbb{N}^{>0}$, consider $n$ subdivisions of $[0,1]$, and the corresponding intervals $\left[c_{k}, c_{k+1}\right)$, where $c_{k+1}-c_{k}=1 / n, 0 \leq k<n$, and $c_{0}=0, c_{n}=1$. If $p=0$, let $a(n)=0$ for each $n \in \mathbb{N}^{>0}$. Otherwise, using the customary covering argument relative to such intervals, the number $p$ is a member of one and only one of these intervals, for each $n \in \mathbb{N}^{>0}$. Hence for each such $n>0$, select the end point $c_{k}$ of the unique interval $\left[c_{k}, c_{k+1}\right)$ that contains $p$. Notice that for $n=1, c_{k}=c_{0}=$ 0 . For each such selection, let $a(n)=k$. Using this inductive styled definition for the sequence $a$, it is immediate, from a simple induction proof, that $a \in A, g_{a p} \in F$, and that $\lim _{n \rightarrow \infty} \mathcal{g}_{a p}(n)=p$. For example, consider the basic sequence $a$ in paragraph 2 of this proof. Then $g_{a p}=\{(1,0),(2,1),(3,1),(4,2),(5,2),(6,3),(7,3),(8,4), \ldots\}$ is such a sequence that converges to $1 / 2$. Let $F_{p} \subset F$ be the nonempty set of all such $g_{a p}$. Note that for the set $F_{p}, p$ is fixed and $F_{p}$ contains each $g_{a p}$, as $a$ varies over $A$, that satisfies the convergence requirement. Thus, for $0 \leq p \leq 1, A$ is partitioned into subsets $A_{p}$ and a single set $A^{\prime}$ such that each member of $A_{p}$ determines a $g_{a p} \in F_{p}$. The elements 
of $A^{\prime}$ are the members of $A$ that are not so characterized by such a $p$. Let $\mathscr{A}$ denote this set of partitions.

Let $B=\left\{f \mid \forall n \forall m\left(\left(\left(n \in \mathbb{N}^{>0}\right) \wedge(m \in \mathbb{N}) \wedge(m \leq n)\right) \rightarrow((f:([1, n] \times\{n\}) \times\{m\} \rightarrow\right.\right.$ $\left.\left.\{0,1\}) \wedge\left(\forall j\left(\left(\left(j \in \mathbb{N}^{>0}\right) \wedge(1 \leq j \leq n)\right) \rightarrow\left(\sum_{j=1}^{n} f(((j, n), n), m)=m\right)\right)\right)\right)\right\}$. The members of $B$ are determined, but not uniquely, by each $(n, m)$ such that $\left(n \in \mathbb{N}^{>0}\right) \wedge$ $(m \in \mathbb{N}) \wedge(m \leq n)$. Hence for each such $(n, m)$, let $f_{n m} \in B$ denote a member of $B$ that satisfies the conditions for a specific $(n, m)$.

For a given $p$, by application of the axiom of choice, with respect to $\mathscr{A}$, there is an $a \in$ $A_{p}$ and a $g_{a p}$ with the properties discussed above. Also there is a sequence $f_{n a(n)}$ of partial sequences such that, when $n>1$, it follows that $(\dagger) f_{n a(n)}(j)=f_{(n-1) a(n-1)}(j)$ as $1 \leq j \leq(n-1)$. Relative to the above example, consider the following:

$$
\begin{gathered}
f_{1 a(1)}(1)=0, \\
f_{2 a(2)}(1)=0, \quad f_{2 a(2)}(2)=1, \\
f_{3 a(3)}(1)=0, \quad f_{3 a(3)}(2)=1, \quad f_{3 a(3)}(3)=0, \\
f_{4 a(4)}(1)=0, \quad f_{4 a(4)}(2)=1, \quad f_{4 a(4)}(3)=0, \quad f_{4 a(4)}(4)=1, \\
f_{5 a(5)}(1)=0, \quad f_{5 a(5)}(2)=1, \quad f_{5 a(5)}(3)=0, \quad f_{5 a(5)}(4)=1, \quad f_{5 a(5)}(5)=0, \ldots
\end{gathered}
$$

It is obvious how this unique sequence of partial sequences is obtained from any $a \in A$. For each $a \in A$, let $B_{a}=\left\{f_{n m} \mid \forall n\left(n \in \mathbb{N}^{>0} \rightarrow m=a(n)\right)\right\}$. Let $B_{a}^{\dagger} \subset B_{a}$ such that each $f_{n m} \in B_{a}^{\dagger}$ satisfies the partial sequence requirement (†). For each $n \in \mathbb{N}^{>0}$, let $P f_{n a(n)} \in B_{a}^{\dagger}$ denote the unique partial sequence of $n$ terms generated by an $a$ and the (†) requirement. In general, as will be demonstrated below, it is the $P f_{n a(n)}$ that yields the set of consequence operators as they are defined on $L_{1}$. Consider an additional map $M$ from the set $P F=\left\{P f_{n a(n)} \mid a \in A\right\}$ of these partial sequences into our descriptive language $L_{1}$ for the source $G$ and events $E, E^{\prime}$ as they are now considered as labeled by the tick marks. For each $n \in \mathbb{N}^{>0}$, and $1 \leq j \leq n$, if $P f_{n a(n)}(j)=0$, then $M\left(P f_{n a(n)}(j)\right)=E^{\prime}$ (i.e., $E^{\prime}=E$ does not occur); if $P f_{\text {na }(n)}(j)=1$, then $M\left(P f_{n a(n)}(j)\right)=E$ (i.e., $E$ does occur), as $1 \leq j \leq n$, where the partial sequence $j=1, \ldots, n$ models the intuitive concept of an event sequence since each $E$ or $E^{\prime}$ now contains the appropriate Kleene "tick" symbols or natural number symbols that are an abbreviation for this tick notation.

Consider the set of consequence operators, each defined on $L, H=\{C(X,\{G\}) \mid$ $X \subset L\}$, where if $G \in Y$, then $C(X,\{G\})(Y)=Y \cup X$; if $G \notin Y$, then $C(X,\{G\})(Y)=Y$. Then for each $a \in A_{p}, n \in \mathbb{N}^{>0}$ and the respective $P f_{n a(n)}$, there exists the set of consequence operators $C_{a p}=\left\{C\left(\left\{M\left(P_{n a(n)}(j)\right)\right\},\{G\}\right) \mid 1 \leq j \leq n\right\} \subset H$. Note that from [3, page 5], $H$ is closed under the finite $\vee$ and the actual consequence operator is $C\left(\left\{M\left(P_{n a(n)}(1)\right)\right\} \cup \cdots \cup\left\{M\left(P_{n a(n)}(n)\right)\right\},\{G\}\right)$. Applying a realism relation $R$ (i.e., in general, $R(C(\{G\}))=C(\{G\})-\{G\})$ to $C\left(\left\{M\left(P_{n a(n)}(1)\right)\right\} \cup \cdots \cup\left\{M\left(P_{n a(n)}(n)\right)\right\}\right.$, $\{G\})(\{G\})$ yields the actual labeled or identified event partial sequence $\left\{M\left(P_{n a(n)}(1)\right)\right.$, $\left.\ldots, M\left(P_{n a(n)}(n)\right)\right\}$.

Now embed the above intuitive results into the superstructure $M_{1}=\langle\mathscr{R}, \in,=\rangle$ which is further embedded into the nonstandard structure ${ }^{*} \mu_{1}=\langle * \mathscr{R}, \in,=\rangle[1,2]$. Let $p \in \mathbb{R}$ be such that $0 \leq p \leq 1$, where $p$ represents a theory predicted (i.e., a priori) probability that an event will occur. Applying a choice function $C$ to $\mathscr{A}$, there is some $a \in A_{p}$ 
such that $g_{a p} \rightarrow p$. Thus ${ }^{*} C$ applied to ${ }^{*} \mathscr{A}$ yields $* a \in{ }^{*} A_{p}$ and ${ }^{*} g_{a p} \in{ }^{*} F_{p}$. Let $v \in{ }^{*} \mathbb{N}$ be any infinite natural number. The hyperfinite sequence $\left\{a_{1}, \ldots, a_{n}, \ldots,{ }^{*} a_{v}\right\}$ exists and corresponds to $\left\{a_{1}, \ldots, a_{n}\right\}$ for any natural number $n \in \mathbb{N}^{>0}$. Also we know that $\operatorname{st}\left({ }^{*} a_{\mu}\right)=p$ for any infinite natural number $\mu$. Thus there exists some internal hyperfinite $P f_{v^{*} a(v)} \in{ }^{*} P F$ with the $*$-transferred properties mentioned above. Since ${ }^{*} \mathbf{H}$ is closed under hyperfinite $\vee$, there is a $P_{p} \in{ }^{*} \mathbf{H}$ such that, after application of the relation ${ }^{*} R$, the result is the hyperfinite sequence

$$
S=\left\{{ }^{*} M\left(P_{v^{*} a(v)}(1)\right), \ldots,{ }^{*} M\left(P_{v^{*} a(v)}(j)\right), \ldots,{ }^{*} M\left(P_{v^{*} a(v)}(v)\right)\right\} .
$$

Note that if $j \in \mathbb{N}$, then we have that ${ }^{*} \mathbf{E}=\mathbf{E}$ or ${ }^{*} \mathbf{E}^{\prime}=\mathbf{E}^{\prime}$ as the case may be.

An extended standard mapping that restricts $S$ to internal subsets would restrict $S$ to $\left\{{ }^{*} M\left(P_{v^{*} a(v)}(1)\right), \ldots,{ }^{*} M\left(P_{v * a}(v)(j)\right)\right\}$, whenever $j \in \mathbb{N}^{>0}$. Such a restriction map models the restriction of $S$ to the natural-world in accordance with the general interpretation given for internal or finite standard objects [2, page 98]. This completes the proof.

3. Distributions. Prior to considering the statistical notion of a frequency (mass, density) function and the distribution it generates, there is need to consider a finite Cartesian product consequence operator. Suppose that we have a finite set of consequence operators $\left\{C_{1}, \ldots, C_{m}\right\}$, where at least one is axiomless, each defined upon its own language $L_{k}$. Define the operator $\Pi C_{m}$ as follows: for any $X \subset L_{1} \times \cdots \times L_{m}$, using the projections $\mathrm{pr}_{k}$, consider the Cartesian product $\operatorname{pr}_{1}(X) \times \cdots \times \mathrm{pr}_{m}(X)$. Then $\Pi C_{m}(X)=C_{1}\left(\operatorname{pr}_{1}(X)\right) \times \cdots \times C_{m}\left(\operatorname{pr}_{m}(X)\right)$ is a consequence operator on $L_{1} \times \cdots \times L_{m}$. If each $C_{k}$ is a finite consequence operator, then $\Pi C_{m}$ is finite. In all other cases, $\Pi C_{m}$ is a general consequence operator. All of these standard facts also hold within our nonstandard structure under $*$-transfer.

A distribution's frequence function is always considered to be the probabilistic measure that determines the number of events that occur within a cell or "interval" for a specific decomposition of the events into various definable and disjoint cells. There is a specific probability that a specific number of events will be contained in a specific cell and each event must occur in one and only one cell and not occur in any other cell.

For each distribution over a specific set of cells, $I_{k}$, there is a specific probability $p_{k}$ that an event will occur in the cell $I_{k}$. Assuming that the distribution does indeed depict physical behavior, we will have a special collection of $g_{a p_{k}}$ sequences generated. For example, assume that we have three cells and the three probabilities $p_{1}=1 / 4$, $p_{2}=1 / 2, p_{3}=1 / 4$ occur in $I_{1}, I_{2}, I_{3}$, respectively. Assume that the number of events to occur is 6 . Then the three partial sequences might appear as follows

$$
\begin{aligned}
& g_{a p_{1}}=\{(1,1),(2,1),(3,1),(4,2),(5,2),(6,2)\}, \\
& g_{a p_{2}}=\{(1,0),(2,1),(3,2),(4,2),(5,2),(6,3)\}, \\
& g_{a p_{3}}=\{(1,0),(2,0),(3,0),(4,0),(5,1),(6,1)\} .
\end{aligned}
$$

Thus after six events have occurred, 2 events are in the first cell, 3 events are in the second cell, and only 1 event is in the third cell. Of course, as the number of events 
continues the first sequence will converge to $1 / 4$, the second to $1 / 2$, and the third to $1 / 4$. Obviously, for any $n \geq 1, g_{a p_{1}}(n)+g_{a p_{1}}(n)+g_{a p_{3}}(n)=n$. Clearly, these required $g_{a p_{i}}$ properties can be formally generated and generalized to any finite number $m$ of cells.

Relative to each factor of the Cartesian product set, all of the standard aspects of Theorem 2.1 will hold. Further, these intuitive results are embedded into the above superstructure and further embedded into our nonstandard structure. Hence, assume that the languages $L_{k}=L_{1}$ and that the standard factor consequence operator $C_{k}$ used to create the product consequence operator is a $C_{a p_{k}}$ of Theorem 2.1. Under the nonstandard embedding, we would have that for each factor, there is a pure nonstandard consequence operator $P_{p_{k}} \in{ }^{*} \mathbf{H}_{k}$. Finally, consider the nonstandard product consequence operator $\Pi P_{p_{m}}$. For $*\left(\left\{\mathbf{G}_{1}\right\} \times \cdots \times\left\{\mathbf{G}_{m}\right\}\right)=\left\{\mathbf{G}_{1}\right\} \times \cdots \times\left\{\mathbf{G}_{m}\right\}, \mathbf{G}_{i}=\mathbf{G}$, this nonstandard product consequence operator yields for any fixed event number $n$, an ordered $m$-tuple, where one and only one coordinate would have the statement $\mathbf{E}$ and all other coordinates the $\mathbf{E}^{\prime}$. It would be these $m$-tuples that guide the proper cell placement for each event and would satisfy the usual requirements of the distribution. Hence, the patterns produced by a specific frequency function for a specific distribution may be rationally assumed to be the result of an application of an ultralogic.

\section{REFERENCES}

[1] R. A. Herrmann, The Theory of Ultralogics Part I, http://www.arxiv.org/abs/math.GM/ 9903081.

[2] _ The Theory of Ultralogics Part II, http://www.arxiv.org/abs/math.GM/9903082.

[3] _ Nonstandard consequence operators, Kobe J. Math. 4 (1987), no. 1, 1-14, http://www.arxiv.org/abs/math.LQ/9911204. MR 89d:03068. Zbl 639.03070.

[4] _ Information theory and consequence operators, C. R. S. Quarterly 36 (1999), 123132.

[5] S. C. Kleene, Mathematical Logic, John Wiley and Sons, New York, 1967. MR 36\#25. Zbl 149.24309.

[6] A. Tarski, Logic, Semantics, Metamathematics. Papers from 1923 to 1938, Oxford Press, 1956. MR 17,1171a. Zbl 075.00702.

Robert A. Herrmann: Mathematics Department, U. S. NAVAl ACAdemy, 572C Holloway RD, ANNAPOLIS, MD 21402-5002, USA

E-mail address: rah@usna.edu 


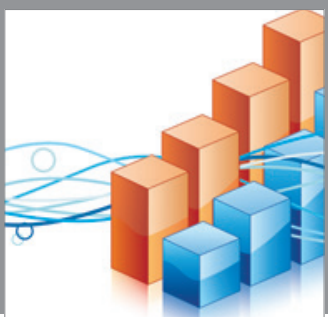

Advances in

Operations Research

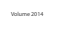

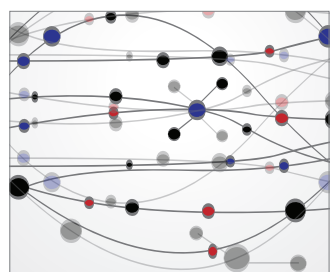

\section{The Scientific} World Journal
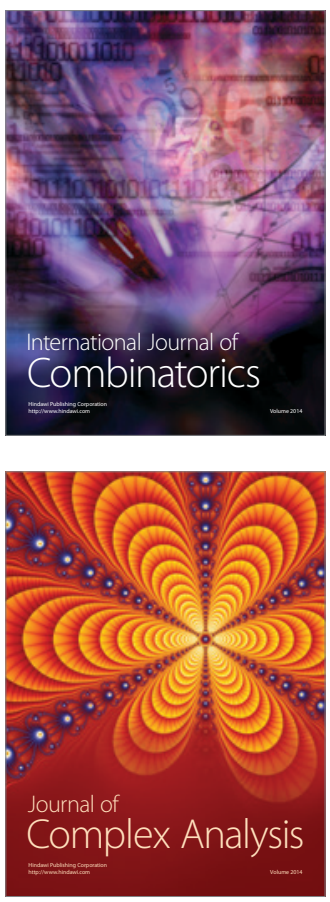

International Journal of

Mathematics and

Mathematical

Sciences
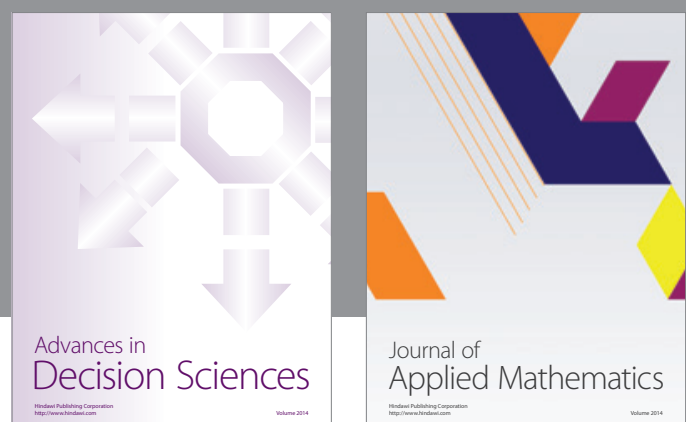

Journal of

Applied Mathematics
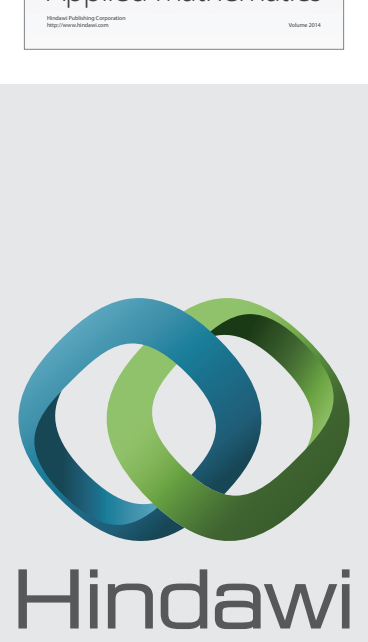

Submit your manuscripts at http://www.hindawi.com
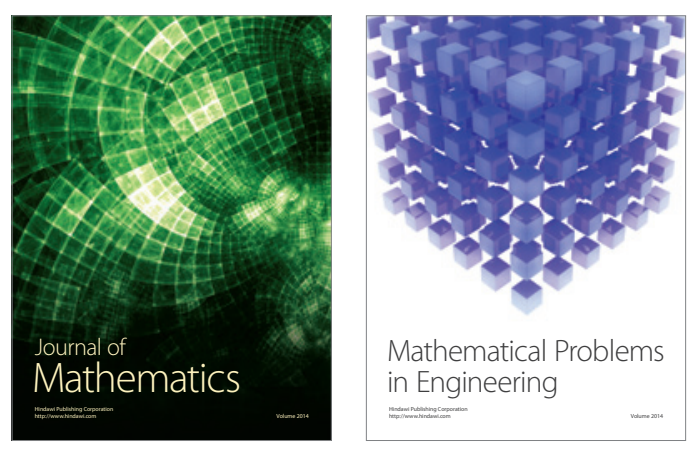

Mathematical Problems in Engineering
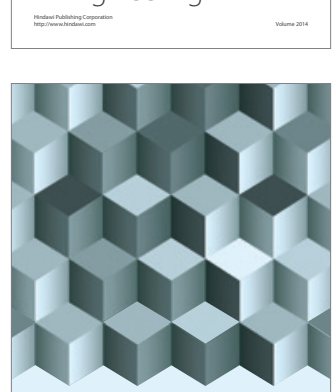

Journal of

Function Spaces
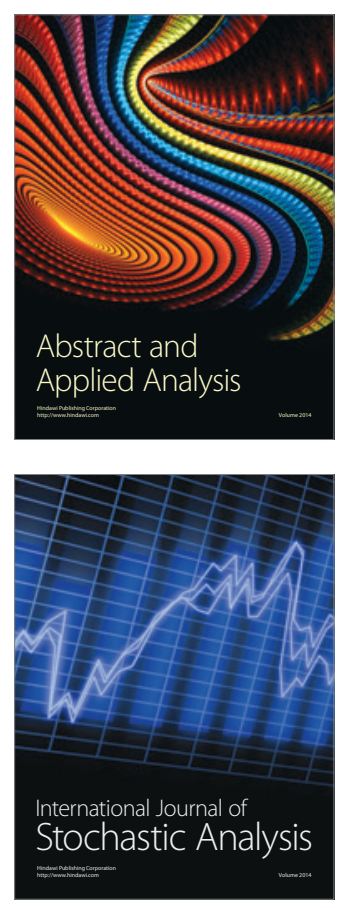

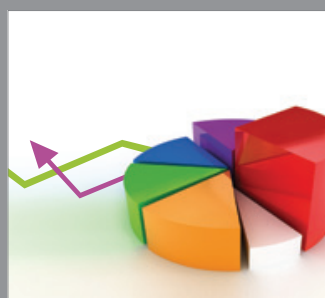

ournal of

Probability and Statistics

Promensencen
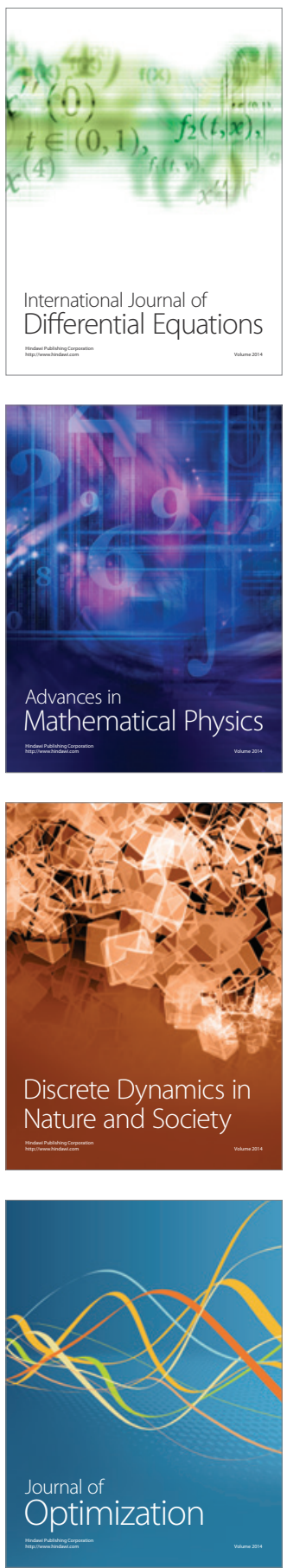